\title{
Imaging techniques with synchrotron radiation
}

\author{
Cyril Petibois
}

Published online: 9 May 2010

(C) Springer-Verlag 2010

Synchrotron radiation (SR) is an electromagnetic radiation generated by the acceleration of ultrarelativistic (i.e. moving close to the speed of light) charged particles through magnetic fields. This is achieved in storage rings, or synchrotrons. Since the discovery of this radiation by Robert Langmuir and Herb Pollock in a General Electric synchrotron accelerator built in 1946, four generations of storage rings have been developed. The first generation, developed in the 1950s, were dedicated high-current accelerators for particle physics $(3-33 \mathrm{GeV}$ ), and they rapidly evolved towards electron-positron colliding-beam machines that were operated so as to provide the highest possible collision rates without blowing up the beams - a condition that provided low beam currents. These storage rings operated in parasitic mode, thus limiting their SR output.

The second generation of storage rings were designed in the early 1970 s to produce SR with exceptional spectral brightness or brilliance, which was achieved by inserting devices such as undulators and wigglers that took maximum advantage of the intrinsic brightness of the SR source. However, this generation of storage rings, with their low emittance and long straight sections for undulators, could not achieve the desired brightness with spatial coherence. The third generation of storage rings (from the 1990s) were specifically designed to optimize SR brightness (then SR was used as a light source), but generally focused on short (hard to soft X-rays) or long (soft X-rays to ultraviolet and infrared radiation) wavelengths. The main advantages of

C. Petibois $(\bowtie)$

Université Victor Segalen Bordeaux 2, CNRS UMR 5248, CBMN,

2 Rue Robert Escarpit,

33600 Pessac-Cedex, France

e-mail: c.petibois@cbmn.u-bordeaux.fr high brightness include spatially resolved information, ranging from X-ray to IR microscopy, better beam stability, as well as longer scanning durations (hours) between injections. The resulting beneficial conditions for utilizing synchrotron light sources for imaging purposes led to major developments in microscopy, including X-ray phasecontrast tomography, X-ray fluorescence mapping, infrared microscopy, and many more, all of which benefitted greatly from the high SR source performance. Finally, the fourth and upcoming generation of storage rings use a hard X-ray (wavelength $<1 \AA$ ) free electron laser (FEL) based on a very long undulator in a high-energy electron linear accelerator to achieve peak SR brightnesses that are several orders of magnitude higher than those previously obtained. Thus, imaging techniques will soon benefit from another major boost in SR brightness, which will enable unprecedented analytical performances.

Second- and third-generation SR facilities have been used to develop high-performance imaging methods that utilise the bright SR source to enhance spatial resolution and/or sensitivity. Many experiments have shown the potential of SR as a powerful source, notably for X-ray applications (diffraction, phase contrast, absorption, fluorescence, ...). On the other hand, other applications of SR have so far failed to realise their potential. For example, infrared microscopy has managed only a small gain in sensitivity through the use of SR compared to a Globar source, and its lateral resolution is not enhanced because of the diffraction limit in the mid-IR interval. This lack of enhancement is due to the physics of the SR source, which is round or oval in shape, a few $\mu \mathrm{m}$ in diameter, but exhibits a change in photon flux from the center to the edges of several orders of magnitude. As imaging requires a homogeneous signal-to-noise ratio between image pixels, applications of SR-FTIR require further technological and/or methodological advances in order to benefit from 
increased SR brightness. There are thus some delays between technological enhancements in SR and the development of dedicated microscopy systems, notably in terms of optics and detectors. This situation is illustrated by the difference in end station development over the course of the history of synchrotron radiation use, which started in the 1950s for shorter (X-ray) wavelengths, and progressed in the 1980s to longer (UV-IR) wavelengths. In other words, whereas X-ray beamlines are coupled to end stations designed by physicists from the synchrotron facility, infrared and UV beamlines are usually coupled to commercial instruments that are designed for conventional sources. As a consequence, efforts are now focused on designing microscopes that will complement the physics of SR, with new optics and detectors, as well as collimating several SR sources (source entries from the ring) onto the same detector for photon flux homogenization.

Another challenge relates to imaging techniques with synchrotron radiation, with new developments being aimed at allowing multimodality on the same sample, such as combining X-ray phase-contrast tomography with elemental mapping of metal ions by X-ray fluorescence. This evolution of SR applications toward multimodality shows that such imaging techniques are now maturing, and offer versatile applications in the health and life sciences as well as the characterisation of natural and synthetic materials. Scientific talks during the 2nd International Conference on Imaging Techniques with Synchrotron Radiation (6-10 November 2009, Sanya, Hainan, P.R. China) indicated the major opportunities offered by these large facilities, from molecular to multimodal imaging, and the promise of nanoscale performance in the future. The Sanya meeting was designed to bring world experts together to help build the user community, foster new scientific applications, and nurture young scientists. Twenty-five oral presentations contributed by 40 delegates from eight countries (including China, Australia, USA, France, Germany, Italy, Japan, and the Netherlands) covered the current and future panorama of high-performance imaging using top-performance instrumentation, optics, sources, and detectors.

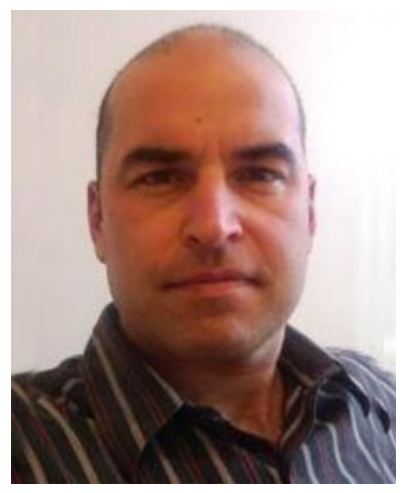

Cyril Petibois is Assistant Professor at the University of Bordeaux 2, France, where he is a biochemistry teacher and a scientist involved in the development of imaging methodology for biosample analysis (CNRS UMR 5248 research unit; "Vibrational spectroscopy group", headed by Dr. Bernard Desbat). C. Petibois' research interest is in the development of multimodal imaging for full characterization of small samples, such as individual cells, combining elemental, morphological, molecular, and chemical techniques. His research unit develops all aspects of the multimodal imaging methodology, i.e. cell cultures dedicated to imaging, post-processing image manipulation for multimodality, specific chemometrics, image functionalization, etc. The laboratory is also engaged in the development of high-performance imaging using synchrotron radiation, notably using FTIR and X-ray beamlines in major European and Asian synchrotron radiation facilities. 